\title{
A Pilot Study for the Establishment of a Bloodsucking Model for Forcipomyia taiwana (Biting Midge)
}

Hung-Yu Shu', Chun-Yao Chen ${ }^{2}$, Ya-Ting Hsu' ${ }^{2}$, Guang-Huey Linn $^{3^{*}}$

${ }^{1}$ Department of Bioscience Technology, Chang Jung Christian University, Tainan, Taiwan

${ }^{2}$ Department of Life Science, Tzu Chi University, Hualien, Taiwan

${ }^{3}$ Department of Microbiology, School of Medicine, Tzu Chi University, Hualien, Taiwan

\section{Abstract}

Background: Forcipomyia taiwana, a small biting midge that has an island-wide presence in Taiwan, is a notoriously annoying bloodsucking pest. Bites from female $F$. taiwana can cause itching, swelling, lesions, and severe anaphylactic reactions; however, little is known about the factors that enhance or hinder their bloodsucking behavior.

Methods: A pilot study of $F$. taiwana was conducted on two New Zealand White rabbits under controlled conditions. Sweat from human subjects was collected and applied to the right ears of study rabbits, while distilled water was applied to the left ears, and differences in F. taiwana feeding behavior were subsequently assessed.

Results: Rabbit ears treated with human sweat experienced greater F. taiwana biting frequency overall, but preliminary results suggest that sweat with higher microbial diversity may induce less feeding behavior. Tentative correlations between bacterial genera and degree of biting attraction to $F$. taiwana were also assessed.

Conclusion: This pilot study of a F. taiwana bloodsucking model provides insight into how human sweat and microbial diversity can affect feeding behavior, and the results may be useful for the development of effective biorepellants against $F$. taiwana. In addition, the rabbit model presented can also serve as a useful platform for future related research.

\section{Introduction}

Forcipomyia taiwana is a tiny $(1.0-1.5 \mathrm{~mm})$ bloodsucking midge that is widely distributed in both urban and rural environments across Taiwan and parts of Southern China [1]. Female F. taiwana are known to feed during the day from the exposed body surfaces of humans, and their bites can cause intense itching, swelling, skin lesions, and even serious anaphylactic reactions; overall, about $60 \%$ of individuals bitten by $F$. taiwana will develop varying degrees of hypersensitivity [2]. Although no midge-borne diseases have been reported in the Taiwanese population as yet, $F$. taiwana bites can be an irritating nuisance at best, or can result in anaphylaxis or cellulitis at worst Therefore, an understanding of the factors that enhance or inhibit $F$. taiwana feeding behavior may be useful to controlling the current and future impact posed by these biting midges.

Recent research has increasingly shown that the feeding behavior of bloodsucking insects is not purely affected by interactions between the insects and human hosts alone; for example, the Plasmodium malaria parasite has been reported to influence the feeding behavior of female Anopheles gambiae mosquitoes [3,4], and studies show that human skin microbiota can affect the feeding preferences of mosquitoes [36], in part by altering body order [3]. However, it is as yet unclear whether $F$. taiwana demonstrates odor-mediated host-seeking and feeding behavior. More importantly, a suitable animal model to examine F. taiwana feeding patterns has not yet been developed. Mice (Mus musculus) have been used as animal models to examine feeding behavior in mosquitoes (Culexpipiens) [7], or to assess the effectiveness of repellants against laboratory-cultured mosquitoes [8]. Unfortunately, due to the differences in proboscis structure between mosquitoes and midges, mice cannot be used as animal models to examine midge feeding behavior, as midges lack the elongated proboscis of mosquitoes and need to be in contact with exposed skin in order to use their mandibles to draw blood. A recent publication midges in humans [1]. presenting the development of a murine model for F. taiwana allergies utilized intradermal application of midge extract, with no actual biting behavior involved [9]. Previously, in order to elucidate the midge life cycle from eggs to pupae, researchers described the use of their own blood to nourish female midges; however, although midge-insensitive humans may be relatively unaffected by repetitive feeding, it has been reported that long-term feeding may induce hypersensitivity to biting

In this study, we describe the use of New Zealand White rabbits as an animal model to investigate $F$. taiwana feeding behavior. New Zealand White rabbits are widely used in animal experiments and can be consistently managed in the lab. In addition, the ears of New Zealand White rabbits stand straight up and only have a short fur coating, conditions that are much more conducive to $F$. taiwana feeding as compared to other laboratory animals. We collected human sweat from two different individuals, one of which exhibited preferred feeding by $F$. taiwana. Sweat from each individual was respectively applied to the right ears of two New Zealand White rabbits, with the left ears serving as controls. We found that $F$. taiwana displayed a distinct feeding preference for rabbit ears to which human sweat had been applied; moreover, sweat from individuals with less diverse skin microbiota appeared to be more favored by female biting midges. The

"Corresponding Author: Dr. Guang-Huey Lin, Department of Microbiology, School of Medicine, Tzu Chi University,Number 701, Section 3, Chung-Yang Road, 97004, Hualien, Taiwan; E-mail: veronica@gms.tcu.edu.tw

Citation: Shu HY, Chen CY,Hsu YT, Lin GH (2017) A Pilot Study for the Establishment of a Bloodsucking Model for Forcipomyia taiwana (Biting Midge). Int J Clin Med Microbiol 2: 118. doi: https://doi.org/10.15344/2456-4028/2017/118

Copyright: @ 2016 Shu et al. This is an open-access article distributed under the terms of the Creative Commons Attribution License, which permits unrestricted use, distribution, and reproduction in any medium, provided the original author and source are credited. 
results indicate that skin microbiota may also influence the feeding behavior of $F$. taiwana, and our findings could have interesting implications for the development of biorepellants against this biting midge.

\section{Methods}

\section{Ethics Statement}

Human subjects were enlisted for the purpose of sweat collection only, and were managed in accordance with the Declaration of Helsinki. Prior informed consent was granted by all human subjects for participation in sweat collection for the purposes of this study. The animal experiments in this study were designed and performed in accordance with the US National Institutes of Health Guide for the Care and Use of Laboratory Animals, $8^{\text {th }}$ Edition (https://grants.nih. gov/grants/olaw/Guide-for-the-Care-and-use-of-laboratory-animals pdf) and the Guide for the Care and Use of Laboratory Animals of Tzu Chi University, Hualien, Taiwan (http://www.lac.tcu.edu. tw/?page_id=474). The protocol was approved by the Institutional Animal Care and Use Committee of Tzu Chi University (Permit Number: 104042). Two New Zealand White rabbits of similar age were bred in the Tzu Chi University Laboratory Animal Center, and were housed in a specific pathogen-free facility with individual cage ventilation systems. Study rabbits had free access to autoclaved water and food, and were maintained at room temperature $\left(\sim 25^{\circ} \mathrm{C}\right)$ on a 12 hour light-dark cycle. Rabbits were allowed to acclimatize for at least one week prior to F. taiwana feeding behavior experiments, and all efforts were made to minimize unnecessary suffering.

\section{Collection of F. taiwana}

Female F. taiwana were collected by the human bait method, as previously described [10]. At least 20 female midges were kept in a 1.5 $\mathrm{cm}$ (diameter) $\times 4.5 \mathrm{~cm}$ (height) glass vial with a volume of $10 \mathrm{ml}$, and the vial was covered with tulle to prevent midges from escaping, but which allowed midges to feed on the ears of study rabbits. A total of five such vials were prepared prior to experiments on midge feeding behavior.

\section{Human sweat collection and midge feeding experiment}

At least $1 \mathrm{ml}$ of sweat was respectively collected from each human subject (A and B) and kept in similar small vials prior to use. Human subject $A$ exhibited preferred feeding by $F$. taiwana, while human subject $B$ did not have such a profile. For midge feeding experiments, $100 \mu \mathrm{l}$ of human sweat from human subjects $\mathrm{A}$ and $\mathrm{B}$ were respectively applied to the right ears of study rabbits $A$ and $B$, while $100 \mu \mathrm{l}$ of $\mathrm{ddH}_{2} \mathrm{O}$ were applied to the left ears. Vials containing female $F$. taiwana midges were then placed over rabbit ears for 30 minutes, and feeding behavior was observed and recorded. Midge-containing vials were then placed at $4^{\circ} \mathrm{C}$ for 20 minutes to rest midges for easy observation, after which feeding outcomes were observed under a microscope. The results were then compared with the real-time observation results.

\section{Total DNA isolation from sweat}

The remaining sweat was transferred from storage vials to microcentrifuge tubes, and centrifuged at 10,000 xg for 10 minutes. Total DNA was isolated according to a previously described method, with minor modifications [11]. Precipitates with bacteria and human skin debris were resuspended in $500 \mu \mathrm{l}$ of Solution I ( $50 \mathrm{mM}$ glucose, $25 \mathrm{mM}$ Tris-Cl (pH 8.0), $10 \mathrm{mM}$ EDTA (pH 8.0)) with lysozymes (1 $\mathrm{mg} / \mathrm{ml}$ ), and kept at $37^{\circ} \mathrm{C}$ for 30 minutes. Afterwards, $100 \mu \mathrm{l}$ of $10 \%$
SDS and $20 \mu \mathrm{l}$ protease $\mathrm{K}(20 \mathrm{mg} / \mathrm{ml})$ were added, and the mixture was incubated at $55{ }^{\circ} \mathrm{C}$ for one hour. An equal volume $(620 \mu \mathrm{l})$ of phenol/chloroform was then added to extract the DNA, which was subsequently precipitated with 0.1 volume of $5 \mathrm{~N} \mathrm{NaCl}$ and 2 volumes of ice-cold $100 \%$ ethanol at $-80{ }^{\circ} \mathrm{C}$ for 20 minutes.

\section{PCR amplification of $16 S$ rDNA}

PCR reactions were performed to amplify $16 \mathrm{~S}$ rDNA, using the following primer pairs as previously described: [12] (i) Bakt341F: 5'-CCTACGGGNGGCWGCAG-3' and Bakt805R: 5'-GACTACHVGGGTATCTAATCC-3'; and (ii) Bakt-341Fad: 5'-TC GTCGGCAGCGTCAGATGTGTATAAGAGACAGCCTACGGGNG GCWGCAG-3' and Bakt-805Rad:

5'-GTCTCGTGGGCTCGGAGATGTGTATAAGAGACAGGAC TACHVGGGTATCTAATCC-3'. Reactions were performed in $50-\mu \mathrm{l}$ volumes containing $250 \mu \mathrm{M}$ dNTP, $0.5 \mu \mathrm{M}$ of each primer, $1.25 \mathrm{U}$ Taq DNA polymerase, $1.25 \mathrm{U}$ Pfu DNA polymerase, and $1 \times$ reaction buffer. PCR conditions were as follows: initial denaturation at $94^{\circ} \mathrm{C}$ for 5 minutes, followed by 20 cycles of denaturation $\left(94^{\circ} \mathrm{C}\right.$ for 30 seconds), annealing $\left(55^{\circ} \mathrm{C}, 30\right.$ seconds), extension $\left(72^{\circ} \mathrm{C}, 30\right.$ seconds $)$, and a final extension step at $72^{\circ} \mathrm{C}$ for 5 minutes. PCR products were analyzed by electrophoresis, and purified using a PCR clean up kit (Geneaid, Taipei, Taiwan), after which $10 \mu \mathrm{l}$ of purified products were used as templates to perform another 5 PCR cycles using the second pair of primers, under the same conditions as that used with the first pair of primers. The quality and quantity of extracted DNA were analyzed by agarose gel electrophoresis, as well as by spectrophotometry using a NanoDrop 2000C spectrophotometer (Thermo Scientific, Waltham, MA).

\section{Metagenomic analysis of microbial diversity in sweat}

A total of 206,191 sequences of 16 S rDNA were analyzed by mothur v1.34.1 software, using the MiSeq standard operational procedure (http://www.mothur.org/wiki/MiSeq_SOP) for defining OTUs (operational taxonomy units). Sequences shorter than 480 bp were removed, and sequences were grouped into OTUs by using the nonredundant (NR) SILVA v119 database with a minimum sequence identity of $97 \%$. Chimeras were examined and removed by using the command: chimera.uchime. Sequences originating from chloroplasts and mitochondria were removed. Finally, 55,671 sequences from the sweat of human subject $\mathrm{A}$ and 47,860 sequences derived from the sweat of human subject B were classified and assigned into OTUs based on SILVA taxonomy. Two communities were randomly subsampled into 47,860 sequences for subsequent community comparison.The representative sequence of each OTU was further classified using the Ribosomal Database Project (RDP) classifier and GenBank database[13]. Microbial diversity was analyzed by PAST v2.17 softwareto determine all diversity indexes.

\section{Results}

New Zealand White rabbits as an animal model for F. taiwana feeding behavior

The suitability of New Zealand White rabbits as an animal model to investigate $F$. taiwana feeding behavior was assessed, with the relatively exposed ears considered to be the most appropriate region for midges to feed upon. Vials containing at least 20 midges were used to perform midge feeding tests. The vials were covered with tulle 
to prevent midges from escaping but still allow feeding to proceed, and these vials were used to cover the ears of study rabbits for 30 minutes. Vials were then placed at $4^{\circ} \mathrm{C}$ to immobilize midges, after which midges were examined under a dissecting microscope to assessfeeding outcomes. Results showed that midges with bloated abdomens engorged with blood were widely observed in the treatment groups (Figure 1), but several midges with flat abdomens and no evidence of feeding were observed in the control groups.

\section{Human sweat induces differential feeding behavior in F. taiwana midges}

Study rabbits $A$ and $B$, the right ears of which were respectively smeared with sweat from human subjects A and B, were used to conduct feeding experiments in the Laboratory Animal Center of Tzu Chi University. Human subject A exhibited preferred feeding by F. taiwana, and during the feeding experiment, more midges were observed on the tulle covering of the vial on the right ear of study rabbit A (Figure 2a), which was smeared with sweat from human subject $A$; however, in other vials, midges were equally distributed around the space (Figure $2 b-d$ ).

After the feeding experiment was conducted for 30 minutes, bite marks were found on both right ears of study rabbits. However, bite marks were larger and more visible on the right ear of study rabbit $A$ as compared to study rabbit $\mathrm{B}$, while no significant bite marks were observed on the left ears of both study rabbits (Figure 3). All bite marks subsequently disappeared within one hour after the feeding experiment was concluded.

After the feeding experiment, $F$. taiwana midges were observed under a dissecting microscope to assess feeding outcomes. Bloated midge abdomens engorged with blood were counted to determine the biting frequency for each vial. The vial on the right ear of study rabbit A had the highest biting frequency observed (Figure 4a, (R)), while the vial on the right ear of study rabbit $B$ (Figure $4 \mathrm{~b},(\mathrm{R})$ ) did not differ greatly from controls (Figure $4 \mathrm{a},(\mathrm{L})$ and $4 \mathrm{~b},(\mathrm{~L})$ ). Considering that the right ear of study rabbit $B$ was smeared with sweat from human subject B, who did not exhibit any preferential feeding by $F$. taiwana, the results suggest that human sweat can induce differential feeding behavior in such biting midges.

\section{High skin microbiota diversity attenuates $F$. taiwana feeding}

To understand the differences in $F$. taiwana feeding behavior induced by the sweat from human subjects A and B, we sought to examine the bacterial populations present in sweat, as this reflects upon the diversity of skin microbiota for these individuals. Total DNA in the sweat from human subjects were isolated and amplified with $16 \mathrm{~S}$ rDNA specific primers for subsequent next-generation sequencing (NGS) analysis. After data filtering processes, a total of 95,720 valid reads and 279 OTUs were obtained from two groups of samples. Altogether, 189 and 210 OTUs were respectively obtained from the sweat samples derived from human subject A (midge-feeding preference) and subject B (non-preference). These sequences were assigned to 19 different phyla (Figure 5a) and 171 different genera (Figure $5 \mathrm{~b}$ ). The Shannon diversity index, a biodiversity measure that incorporates both richness and evenness, was also calculated for the sweat samples. The Shannon diversity index for human subject $B$ (1.866) was higher than that for human subject A (1.077), suggesting that $F$. taiwana midges may be attracted to humans with lower skin microbiota diversity.

Bacteria of the Firmicutes phyla predominated in the sweat sample from human subject A, followed by Proteobacteria and Actinobacteria; however, bacteria from these three phyla were distributed equally in the sweat sample from human subject B (Figure 5a). Moreover, Staphylococcus made up $80 \%$ of the genera in the sweat sample from subject $A$, but represent less than $50 \%$ of the genera in the sample from subject B (Figure 5b), suggesting that the abundance of Staphylococcus spp. may correlate with the relative attractiveness of human subject A to F. taiwana. In addition, Serratia and Propionibacteria respectively
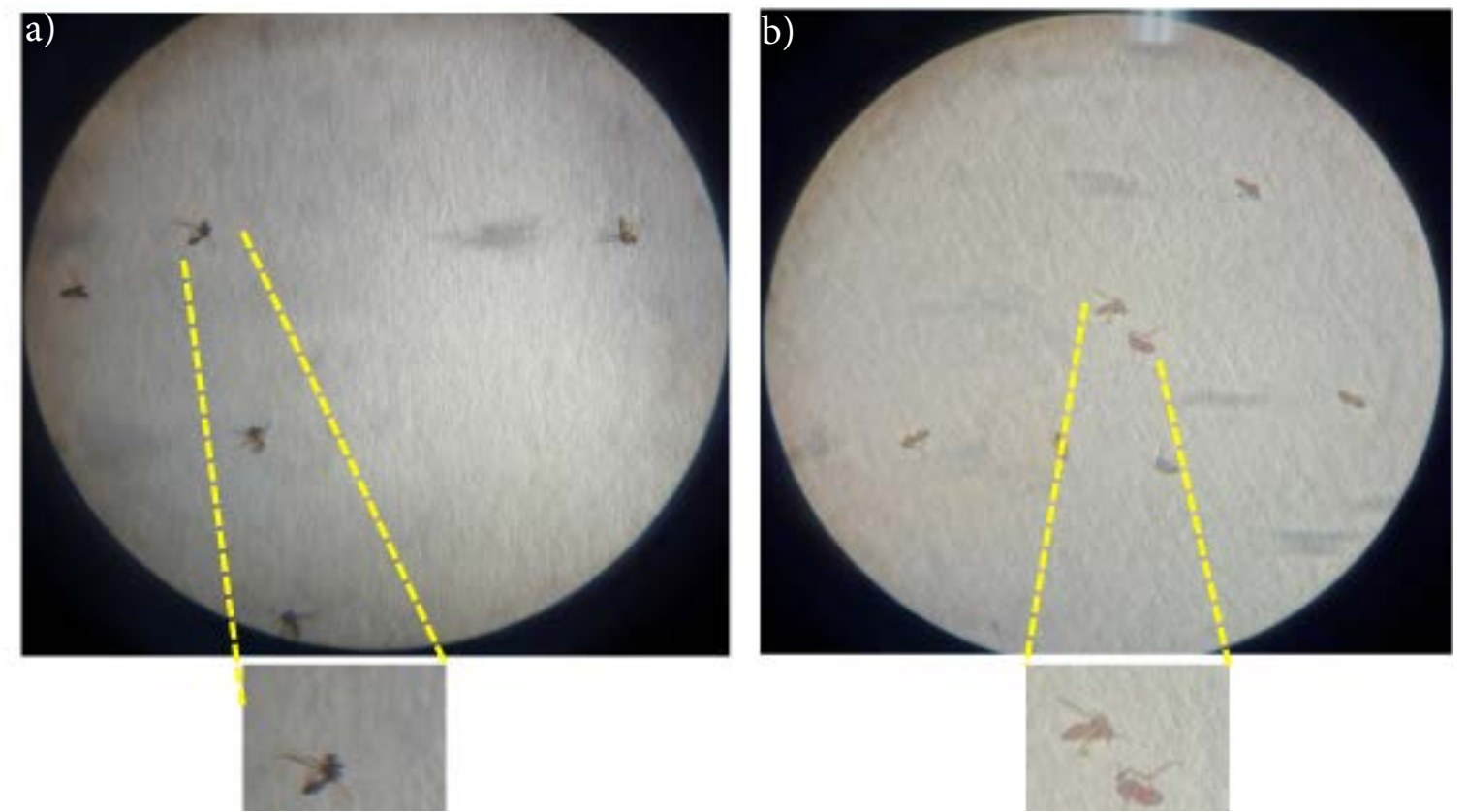

Figure 1: F. taiwana feeding behavior on New Zealand White rabbits, as examined under a dissecting microscope. (A) Midges before the feeding experiment. (B) Midges after feeding on the ears of New Zealand White rabbits. 
Citation: Shu HY, Chen CY,Hsu YT, Lin GH (2017) A Pilot Study for the Establishment of a Bloodsucking Model for Forcipomyia taiwana (Biting Midge). Int J Clin Med Microbiol 2: 118. doi: https://doi.org/10.15344/2456-4028/2017/118

a)

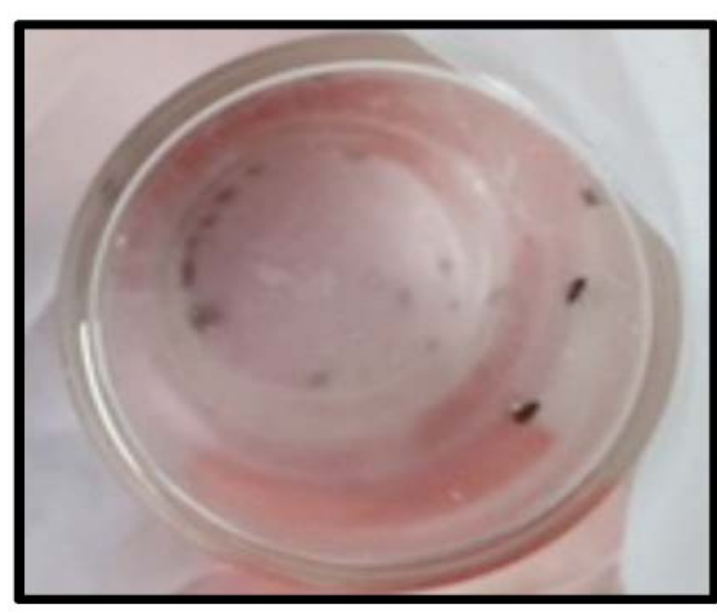

c)

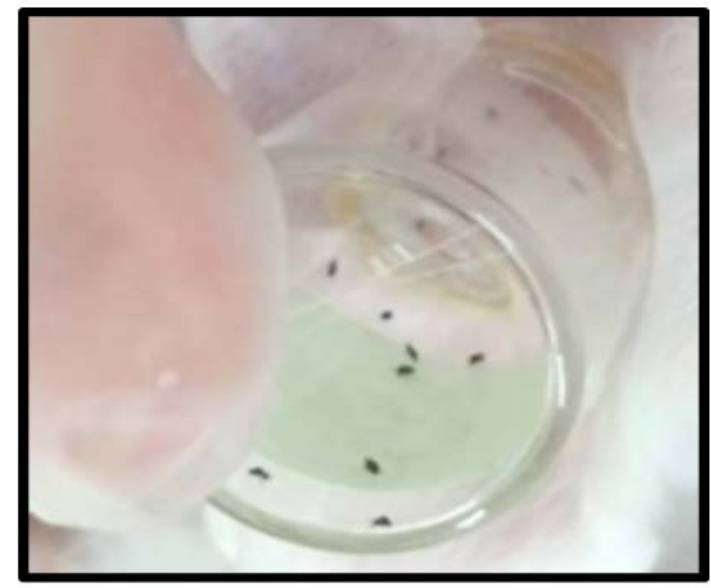

b)

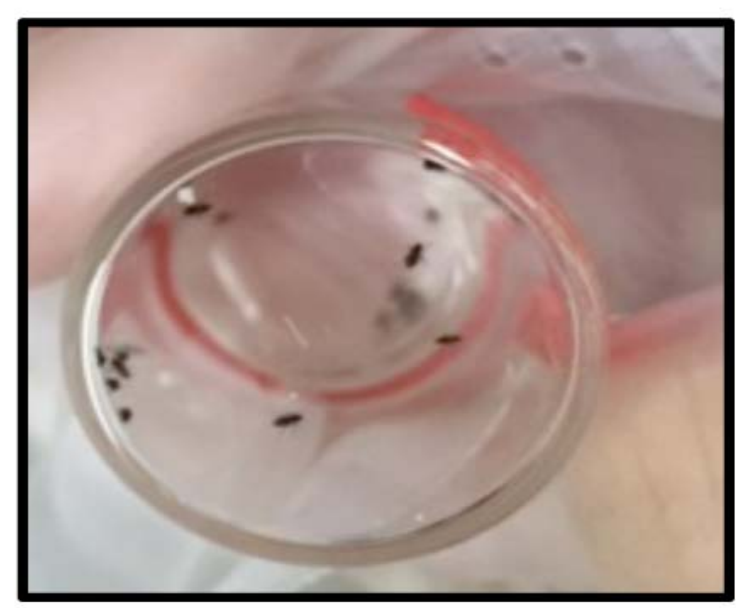

d)

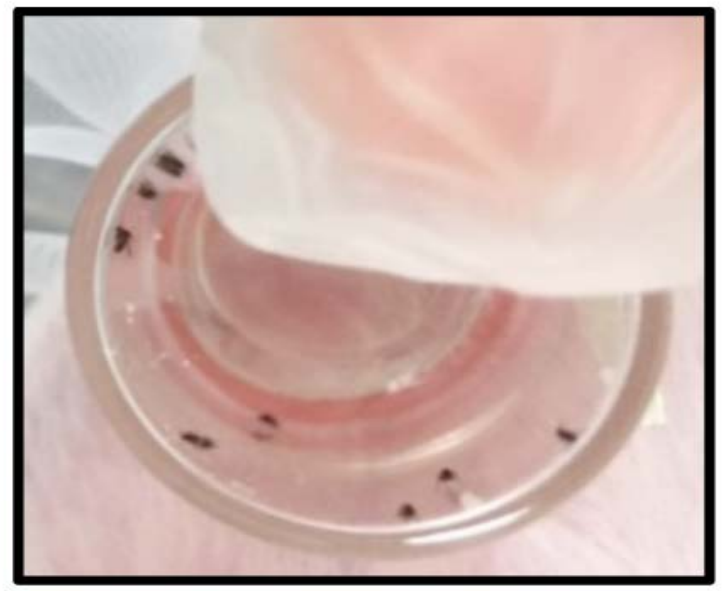

Figure 2: F. taiwana distribution in vials placed over the ears of New Zealand White rabbits during feeding experiments. Glass vials on (A) the right ear and (B) the left ear of study rabbit A; the right ear was smeared with sweat from human subject A, while the left ear was smeared with $\mathrm{ddH}_{2} \mathrm{O}$ as a control. Glass vials on (C) the right ear and (D) the left ear of study rabbit B; the right ear was smeared with sweat from human subject B.

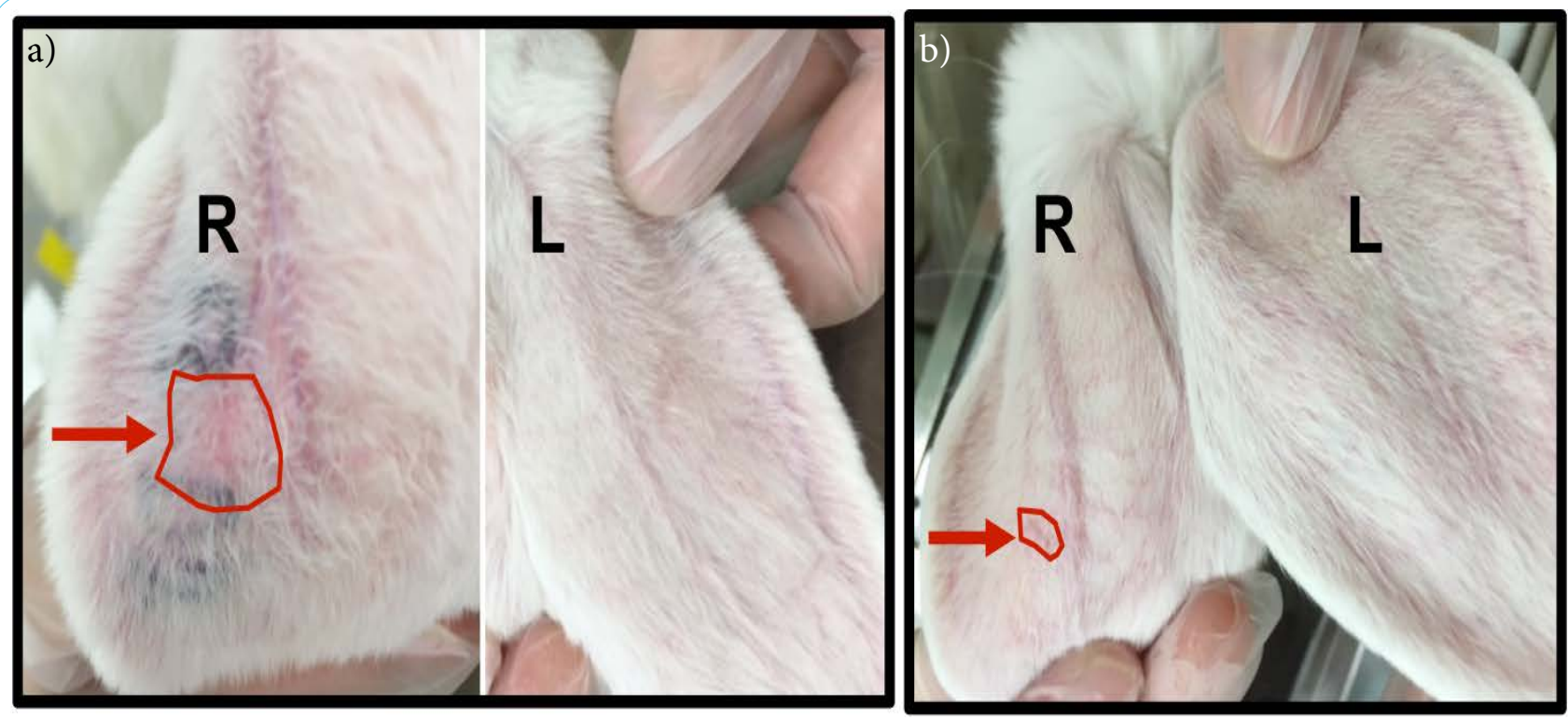

Figure 3: F. taiwana bite marks on the ears of study rabbits. The right (R) and left (L) ears of (A) study rabbit A and (B) study rabbit B are shown. Bite marks are highlighted with red lines and arrows. 
make up $19.6 \%$ and $11.5 \%$ of the skin microbiota of subject B (Figure $5 b)$, suggesting that these two genera may exert a repelling effect. By contrast, Streptococcus, Neisseria, Derxia, Capnocytophaga and Rothia were present in the sweat of subject $A$ at levels 10-fold higher than subject $B$, suggesting that these genera may have an attractant effect for F. taiwana.

\section{Discussion}

There are currently no reports that $F$. taiwana acts as a disease vector, but their bites do pose a risk of hypersensitivity and anaphylaxis [10]. A murine model of $F$. taiwana bite allergy has recently been

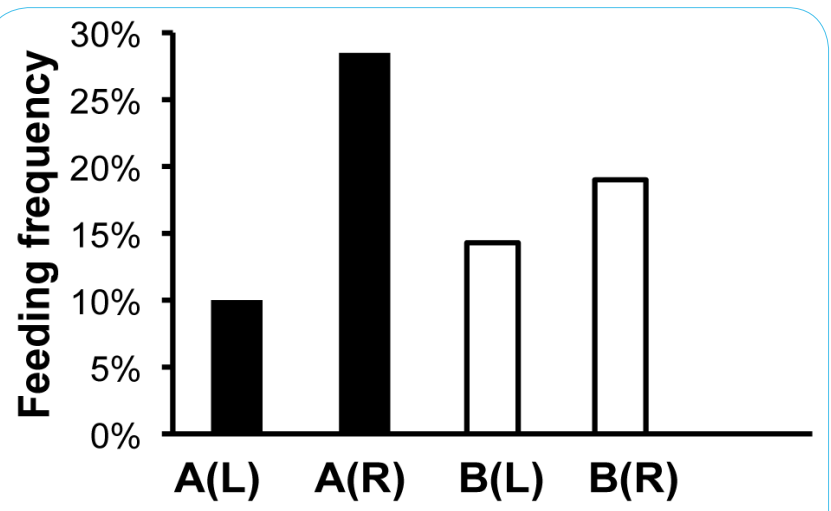

Figure 4: Feeding frequency of $F$. taiwana on the ears of study rabbits. Feeding outcomes on the right $(\mathrm{R})$ and left (L) ears of (A) study rabbit A and (B) study rabbit $\mathrm{B}$ are shown. established, using a two-step sensitization protocol that involved the intraperitoneal injection of mice with $F$. taiwana whole body extract to study the immunopathologic features of midge allergy [9]. However, extracts cannot fully replicate the feeding behavior of $F$. taiwana, and thus a suitable animal model is needed to investigate the life cycle and potential disease transmission capability of $F$. taiwana in more detail. In this study, we describe just such an animal model, using New Zealand White rabbits. This model offers researchers an opportunity to examine the natural habits and behavior of $F$. taiwana under controlled conditions, and without having to rely on human sources for feeding.

It is currently known that the host-seeking behavior of mosquitoes is affected by diverse factors, including $\mathrm{CO}_{2}$ exhalation, host body odor, human leukocyte antigen (HLA) profiling or even beer consumption[14]. Carbon dioxide is a major constituent of exhaled breath, and appears to be an attractant for some mosquitoes; however, carbon dioxide is not a major attractant for the Afrotropical female malaria mosquito, Anopheles gambiae [15], which prefers to exploit olfactory signals emanating from human hosts[16]. Malaria mosquitoes have been reported to be attracted to compounds such as lactic acid, 2-methylbutanoic acid, tetradecanoic acid, and octanal in human body odor[17], and recent studies have assayed 300-400 compounds in human odor with gas chromatography (GC)/mass spectrometry (MS) to identify possible attractants for the Aedes aegypti mosquito[18]; however, further bioassays will be needed to derive definitive conclusions. Nevertheless, it is known that the volatiles that make up human odor are mostly derived from the secretions of skin glands, which are originally odorless to the human nose, but

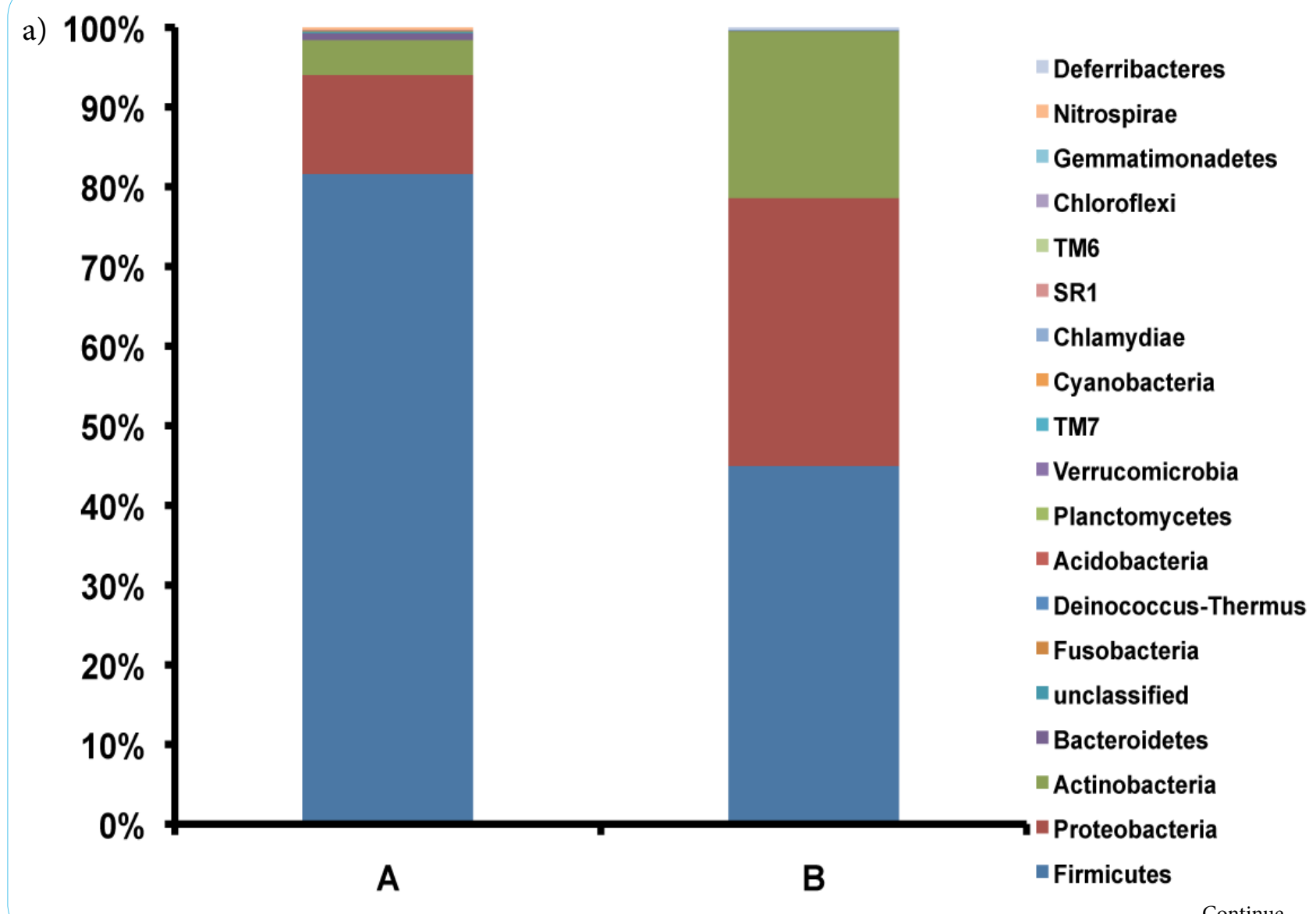




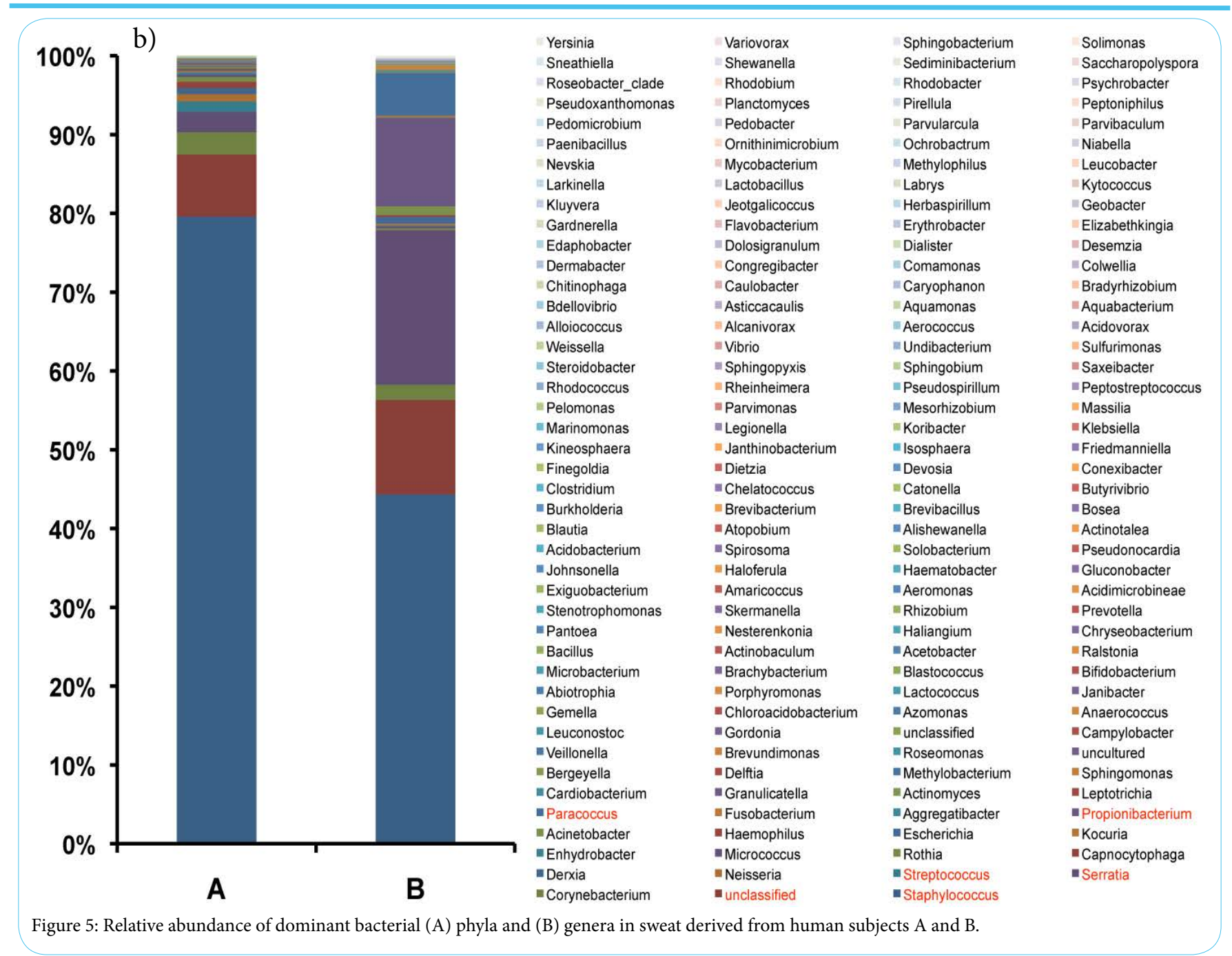

are subsequently converted into odorous and volatile compounds through the enzymatic action of skin microbiota. The body odor of individual humans has been correlated with the presence of specific microorganisms [19]; for example, culture-based studies have shown that human skin microbiota is dominated by corynebacteria staphylococci, or propionibacteria, of which corynebacteria have been associated with the production of odorous steroids and thioalcohols, while staphylococci are known to metabolize branched aliphatic amino acid to short-chain (C2-C5) volatile fatty acids (VFAs) [6]. In this study, we show that midges are attracted to sweat from an individual with a skin microbiota dominated by staphylococci ( $\sim 80 \%)$, and this raises the possibility that VFAs may be an important attractant for F. taiwana; however, further GC/MS analysis of volatile compounds in sweat and an expansion of the current pilot study to include more individuals would be needed to confirm this.

In summary, we showed that New Zealand White rabbits may serve as an animal model to examine feeding behavior for $F$. taiwana, and also presented preliminary results from a pilot study that demonstrated human sweat may influence $F$. taiwana feeding preferences. Our findings suggest that skin microbiota diversity and the presence of specific microorganisms may also play a role in making an individual more or less susceptible to $F$. taiwana biting, and may have implications for the development of effective biorepellants against biting midges in future.

\section{Competing Interests}

The author declares that the has no competing interests.

\section{Acknowledgments}

The authors would like to thank Dr. Hsien-Ming Lee from the Institute of Medical Biotechnology, Central Taiwan University of Science and Technology, and Dr. Ming-Hseng Wang from the Laboratory Animal Center of Tzu Chi University for technical advice. The authors also would like to thank Miss Yu-Hsuan Chiu, Meng-Yun Wen and Mr. Kun-Sheng Hsieh for helping to conduct midge feeding experiments.

\section{Funding}

This study was supported by a grant from the Ministry of Science and Technology, Taiwan (MOST104-2320-B-320-011).

\section{References}

1. Yeh CC, Chuang YY (1996) Colonization and bionomics of Forcipomyia taiwana (Diptera:Ceratopogonidae) in the laboratory. J Med Entomol 33 : 445-448.

2. Chen YH, Lee MF, Lan JL, Chen CS, Wang HL, et al. (2005) Hypersensitivity to Forcipomyia taiwana (biting midge): clinical analysis and identification of major For $t 1$, For $t 2$ and For $t 3$ allergens. Allergy 60: 1518-1523. 
Citation: Shu HY, Chen CY,Hsu YT, Lin GH (2017) A Pilot Study for the Establishment of a Bloodsucking Model for Forcipomyia taiwana (Biting Midge). Int J Clin Med Microbiol 2: 118. doi: https://doi.org/10.15344/2456-4028/2017/118

Page 7 of 7

3. Braks MA, Anderson RA, Knols BG (1999) Infochemicals in mosquito host selection: human skin microflora and Plasmodium parasites. Parasito Today 15: 409-413.

4. Cator LJ, Lynch PA, Thomas MB, Read AF (2014) Alterations in mosquito behaviour by malaria parasites: potential impact on force of infection. Malar J 13: 164.

5. Verhulst NO, Qiu YT, Beijleveld H, Maliepaard C, Knights D, et al. (2011) Composition of human skin microbiota affects attractiveness to malaria mosquitoes. PLoS One 6:e28991.

6. James AG, Austin CJ, Cox DS, Taylor D, Calvert R (2013) Microbiological and biochemical origins of human axillary odour. FEMS Microbiol Ecol 83 527-540.

7. Abdel-Hamid YM, Wahba MM (2006) Detection of haematologic effects of mosquito biting using an animal model. J Egypt Soc Parasitol 36: 937-944

8. Kongkaew C, Sakunrag I, Chaiyakunapruk N, Tawatsin A (2011) Effectiveness of citronella preparations in preventing mosquito bites: systematic review of controlled laboratory experimental studies. Trop Med Int Health 16: 802-810.

9. Lee MF, Yang KJ, Wang NM, Chiu YT, Chen PC, et al. (2014) The development of a murine model for Forcipomyia taiwana (biting midge) allergy. PLoS One 9: e91871.

10. Chen YH, Lee MF, Tsai JJ, Wu HJ, Hwang GY (2009) Specific lgE and IgC responses and cytokine profile in subjects with allergic reactions to biting midge Forcipomyia taiwana. Int Arch Allergy Immunol 150: 66-74.

11. Lin GH, Chen HP, Huang JH, Liu TT, Lin TK, et al. (2012) Identification and characterization of an indigo-producing oxygenase involved in indole 3-acetic acid utilization by Acinetobacter baumannii. Antonie Van Leeuwenhoek 101: 881-890.

12. Klindworth A, Pruesse E, Schweer T, Peplies J, Quast C, et al. (2013) Evaluation of general $16 S$ ribosomal RNA gene PCR primers for classical and next-generation sequencing-based diversity studies. Nucleic Acids Res 41:e1.

13. Wang Q, Garrity GM, Tiedje JM, Cole JR (2007) Naive Bayesian classifier for rapid assignment of rRNA sequences into the new bacterial taxonomy. Appl Environ Microbiol 73: 5261-5267.

14. Lefèvre T, Gouagna LC, Dabiré KR, Elguero E, Fontenille D, et al. (2010) Beer consumption increases human attractiveness to malaria mosquitoes. PLoS One 5: e9546.

15. Mboera LEG, Knols BGJ, Braks MAH, Takken W (2000) Comparison of carbon dioxide-baited trapping systems for sampling outdoor mosquito populations in Tanzania. Med Vet Entomol 14: 257-263.

16. Takken W, Knols BG (1999) Odor-mediated behavior of Afrotropical malaria mosquitoes. Annu Rev Entomol 44: 131-157.

17. Verhulst NO, Beijleveld H, Qiu YT, Maliepaard C, Verduyn W, et al. (2013) Relation between HLA genes, human skin volatiles and attractiveness of humans to malaria mosquitoes. Infect Genet Evol 18: 87-93.

18. Bernier UR, Kline DL, Barnard DR, Schreck CE, Yost RA (2000) Analysis of human skin emanations by gas chromatography/mass spectrometry. 2 . Identification of volatile compounds that are candidate attractants for the yellow fever mosquito (Aedes aegypti). Anal Chem 72: 747-756.

19. Taylor D, Daulby A, Grimshaw S, James G, Mercer J, et al. (2003) Characterization of the microflora of the human axilla. Int $\mathrm{J}$ Cosmet $\mathrm{S} c$ 25: $137-145$ 